\title{
Topologies induced by groups of characters
}

\author{
W. W. Comfort and K. A. Ross * (Rochester)
}

It is a consequence of the Pontryagin duality theorem that a dense subgroup $H$ of a compact Abelian group $G$ carries the topology induced by the continuous characters on $H$. By a theorem of Weil (see 1.1 and 1.2 below), a topological group is a dense subgroup of a compact group if and only if it is totally bounded. In section 1 we exploit these observations; that is, by studying groups of continuous characters we derive a number of results about totally bounded Abelian groups. Similarities between compact and totally bounded Abelian groups are to be expected; the differences recorded in 1.6 and 1.7 are striking.

Our point of view allows us to give in section 2 a simplified proof of a slight generalization of the following theorem, proved in [6] by Kertész and Szele. Every infinite Abelian group can be topologized in such a way that it is a first countable (equivalently, metrizable) topological group. In section 3 , we use the techniques of section 1 to study and characterize the totally bounded group topologies on the integers.

For another investigation of non locally compact topological Abelian groups and their homomorphisms, we refer the reader to Hejcman [3].

1. Totally bounded groups. The topological groups $(G, \mathfrak{C})$ to be considered in this paper will be Abelian and Hausdorff. A subset $B$ of $G$ is said to be bounded if for each neighborhood $V$ of the identity there is a finite subset $F$ of $G$ for which $B \subset \bigcup x P$. The group $(G, \mathcal{G})$ is said to be locally bounded provided that $G$ contains a bounded nonvoid open set and totally bounded $\left(^{(}\right)$if $G$ itself is bounded.

* The authors were supported in part by the National Science Foundation, under contract NSF-G23799.

( ${ }^{1}$ The group $(G, \mathcal{G})$, if not compact, necessarily admits various distinct compatible uniformities. We restrict our attention here to the so-called left uniformity, consisting of all sets $\left\{(x, y) \in G \times G: x^{-1} y \in U\right\} \quad(U \in \mathcal{C})$. Expressions like "totally bounded" and "Cauchy net", when they occur in this paper, always refer to this uniformity (which, because $G$ is Abelian, coincides with the right uniformity for $G$ ). 
Evidently every subgroup of a compact (resp. locally compact) group is totally bounded (resp. locally bounded). That no other such groups exist is the content of the following theorem of A. Weil.

1.1. THEOREM (Weil [7]). Every locally bounded group $G$ is topologically isomorphic with a dense subgroup of a locally compact group $\vec{G}$, which is unique up to a topological isomorphism leaving $G$ fixed pointwise. If $G$ is totally bounded, then $\bar{G}$ is compact.

It is a well-known theorem (see, for example, 22.17 of [4]) that the family of continuous characters on a locally compact Abelian group separates points. We note in passing that from Weil's theorem it follows that the same assertion is true for a locally bounded Abelian group. For an even more extensive class of topological groups with sufficiently many continuous characters, the reader is referred to [1]

Notation. The group of continuous characters on the topological group $(G, \mathcal{C})$ will be denoted by the symbol $(G, \mathcal{G})^{\wedge}$. The symbol $G_{d}$ will denote the group $G$ endowed with the discrete topology; thus $\left(G_{d}\right)^{\wedge}$ consists of all characters on $G$.

For any point-separating subgroup $\mathfrak{H}$ of $\left(G_{d}\right)^{\wedge}$, we let $\mathcal{G}_{\mathcal{X}}$ be the topology induced on $G$ by $\mathcal{H}$. It is easy to see that $\left(G, \zeta_{\mathcal{H C}}\right)$ is a topological group. A basis at the identity consists of all sets

$$
U(\mathcal{F}, \varepsilon)=\left\{x \in G:|\chi(x)-1|<\varepsilon \text { for all } \chi \in \mathcal{F}^{\mathfrak{F}}\right\},
$$

where $\varepsilon>0$ and $\mathcal{F}$ is a finite subset of $\mathcal{H}$.

1.2. THEOREM. Let $(G, \zeta)$ be an Abelian topological group and let $\mathfrak{H}=(G, \mathfrak{C})^{\wedge}$. Then the following assertions are equivalent:

(a) $(G, \mathcal{G})$ is totally bounded;

(b) $(G, \mathcal{C})$ can be embedded in a compact group $\bar{G}$;

(c) $\mathfrak{C}=\mathfrak{C}_{\mathfrak{H}}$.

Proof. (a) $\Rightarrow(\mathrm{b})$. This is 1.1 .

(b) $\Rightarrow$ (c). Each element in $\mathcal{H}$ is the restriction to $G$ of exactly one element of $(\bar{G})^{\wedge}$. By Pontryagin's duality theorem (24.3 in [4]), the topology on the compact group $\bar{G}$ is that induced by the family $(\bar{G})^{\wedge}$. Hence the topology $G$ on $G$ is that induced by $\mathcal{H}$.

$(c) \Rightarrow$ (a). To show that $\left(G, \mathfrak{G}_{\mathcal{H}}\right)$ is totally bounded, we choose a basic open set $U(\mathcal{F}, \varepsilon)$ in $G$. Let $\mathscr{F}=\left\{\chi_{1}, \ldots, \chi_{m}\right\}$ and find an integer $N$ for which $\left|e^{2 \pi i / N}-1\right|<\varepsilon / 2$. For each $m$-tuple $a=\left(k_{1}, \ldots, k_{m}\right)$ of integers $\left(0 \leqslant k_{j} \leqslant N-1\right)$, consider the set

$$
X_{a}=\left\{x \in G:\left|\chi_{j}(x)-e^{2 \pi i k_{j} / N}\right|<\varepsilon / 2 \text { for } 1 \leqslant j \leqslant m\right\} .
$$

Whenever $X_{a}$ is nonvoid, we choose a point $x_{a}$ in $X_{a}$. Then it is easy to see that

$$
G \subset \bigcup_{a} x_{a} \cdot U(\Im, \varepsilon)
$$

In fact, for each $y$ in $G$, there is an $m$-tuple $\alpha=\left(k_{1}, \ldots, k_{m}\right)$ for which

$$
\left|\chi_{j}(y)-e^{2 \pi i i_{j} / N}\right|<\varepsilon / 2 \quad(1 \leqslant j \leqslant m) .
$$

The theorem above is a statement about point-separating groups of characters of the form $(G, \mathcal{C})^{\wedge}$, where $(G, \mathcal{C})$ is a totally bounded topological group. The following theorem shows that, in fact, every pointseparating group of characters has this form.

1.3. Theorem. If $G$ is an Abetian group and $\mathcal{H}$ is any point-separating group of characters on $G$, then $\left(G, \mathcal{C}_{\mathcal{H e}}\right)^{\wedge}=\mathfrak{H}$.

Proof. It is clear that $\mathscr{H C}\left(G, G_{\mathcal{F e}}\right)^{\wedge}$.

Consider a character $\Psi$ in $\left(G, \mathscr{G}_{\mathcal{J}}\right)^{\wedge}$. Let $\tau$ be the homomorphism of $G$ into the torus $T^{\mathcal{H}}$ defined by $(\tau(x))_{\chi}=\chi(x)$. Since $H_{\text {separates points, }}$ the function $\tau$ is one-to-one. Hence the function $\Theta$ on $\tau(G)$ defined by

$$
\Theta(\tau(x))=\Psi(x)
$$

is well defined. A routine argument shows that $\Theta$ is a continuous character on $\tau(G)$. Being therefore uniformly continuous, the character $\Theta$ can be extended to a continuous character on $[\tau(G)]^{-}$. This character, in turn, can be extended to a continuous character on $T^{\text {He }}$ (see 24.12 of [4]), which we again denote by $\Theta$. By 23.21 of [4] there are elements $\chi_{1}, \ldots, \chi_{n}$ of He and continuous characters $\theta_{1}, \ldots, \theta_{n}$ on $T$ such that

$$
\Theta\left(\left(t_{x}\right)\right)=\prod_{k=1}^{n} \theta_{k}\left(t_{x_{k}}\right) \quad \text { whenever } \quad\left(t_{x}\right) \in T^{\mathfrak{H C}} .
$$

For each $k=1, \ldots, n$ there is an integer $m_{k}$ such that $\theta_{k}(t)=t^{m_{k}}$ for each $t$ in $T$. Hence for each $x$ in $G$ we have

$$
\Psi(x)=\Theta(\tau(x))=\Theta((\chi(x)))=\prod_{k=1}^{n} \theta_{k}\left(\chi_{k}(x)\right)=\prod_{k=1}^{n}\left[\chi_{k}(x)\right]^{m_{k}}
$$

Thus $\Psi=\prod_{k=1}^{n} \chi_{k}^{m_{k}}$, so that $\Psi$ belongs to $\mathcal{H e}$.

1.4. CoRoLlary. Let $G$ be an Abelian group and let $\mathcal{H}_{1}$ and $\mathcal{H}_{2}$ be point-

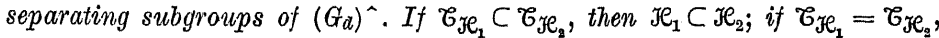
then $\mathfrak{H}_{1}=\mathcal{H e}_{2}$.

1.5. COROLLARY. If Te is a point-separating group of characters on an Abetian group $G$, then $\left(G, \mathcal{G}_{\mathcal{H}}\right)$ is totally bounded.

Proof. Use 1.3 and the implication (c) $\Rightarrow$ (a) of 1.2 .

The following theorem has an analogue valid for general uniform spaces. We treat the special case to which our techniques are readily adapted. 
1.6. THEOREM. Let $\left\{\mathcal{G}_{a}\right\}_{a \in A}$ be a family of topologies on an Abelian group $G$, for each of which $\left(G, \mathcal{C}_{a}\right)$ is a totally bounded topological group. If $\mathcal{G}$ is the smallest topology containing each $\mathcal{C}_{a}$, then $(G, \mathcal{C})$ is a totally bounded topological group.

Proof. The straightforward pronf that $(G, \mathscr{C})$ is a topological group is omitted.

For each $\alpha$ in $A$, we set $\mathscr{H}_{\alpha}=\left(G, \mathscr{C}_{a}\right)^{\wedge}$. Letting $\mathscr{H}$ be the subgroup of $\left(G_{d}\right)^{\wedge}$ generated by $\cup \mathcal{H}_{a}$, one may easily verify that $\mathcal{C}=\mathcal{G}_{\mathcal{J}}$. The conclusion now follows from 1.5.

1.7. THEOREM. For any infinite Abelian group $G$, there is a unique (nondiscrete) totally bounded group topology $\zeta$ such that every homomorphism. of $G$ into a totally bounded group is continnous. In fact, $C=\zeta_{X}$ where $r=\left(G_{d}\right)^{\wedge}$.

Proof. Let $\mathfrak{K}=\left(G_{d}\right)^{\wedge}$ and $\mathscr{C}=\mathscr{C}_{\mathfrak{H e}}$. We first show that a homomorphism $f$ from $(G, \mathcal{C})$ into an arbitrar'y totally bounded group $H$ is continuous. For every continuous character $\chi$ on $H$, it is obvious that the character $\chi \circ f$ is continuous on $(G, \mathfrak{C})$. The continuity of $f$ now follows from the fact that the topology on $H$ is that induced by its continuous characters.

To establish the uniqueness of $\mathscr{C}$, we consider any totally bounded group topology $\mathcal{C}^{\prime}$ on $G$ different from $\mathcal{C}$. Then $\mathcal{C}^{\prime} \subsetneq \mathcal{C}$, so the identity map is a discontinuous homomorphism from $\left(G, \mathfrak{C}^{\prime}\right)$ onto $(G, \mathfrak{C})$.

1.8. Discussion. If in 1.6 the words "totally bounded" are replaced throughout by the word "compact", then (except in the trivial case in which the $\mathfrak{C}_{a}$ 's all coincide) the conclusion must fail. For otherwise the compact topology $\mathfrak{C}$ would properly contain some Hausdorff topology $\mathcal{C}_{a}$, which is impossible.

Theorem 1.7 also gives rise to a distinction between totally bounded and compact groups. Indeed, every infinite compact Abelian group $(G, \smile)$ admits a discontinuous homomorphism into the circle group; that is, $(G, \mathcal{C})^{\wedge} \subsetneq\left(G_{d}\right)^{\wedge}$. To see this, we recall from [5] Kakutani's identity $\operatorname{card}\left(G_{d}\right)^{\wedge}=2^{\operatorname{card}(G)}$. Applying this identity also to the discrete group $(G, \mathcal{C})^{\wedge}$ we obtain the inequalities

$$
\operatorname{card}(G, \mathfrak{C})^{\wedge}<\operatorname{card}(G)<\operatorname{card}\left(G_{d}\right)^{\wedge} .
$$

In corollary 1.4 we gave a one-to-one order-preserving correspondence between totally bounded group topologies for an arbitrar'y (Abelian) group $G$ and point-separating subgroups of $\left(G_{d}\right)^{\wedge}$. Te now characterize these subgroups of $\left(G_{d}\right)^{\wedge}$.

1.9. THEOREM. Let $G$ be an Abelian group and let re be a subgroup of $\left(G_{a}\right)^{\wedge}$. Then $\mathcal{H C}^{2}$ is point-separating if and only if Je is dense in the compact group $\left(G_{d}\right)^{\wedge}$.
Proof. For a family $\mathcal{T}$ of characters, we set

$$
A(\mathcal{F})=\{x \in G: \Psi(x)=1 \text { for each } \Psi \text { in } \mathscr{F}\} .
$$

Theorem 24.10 of [4] states that

$$
\mathfrak{J C}^{-}=\left\{\chi \in\left(G_{d}\right)^{\wedge}: \chi\left(A\left(\mathcal{J C}^{-}\right)\right)=1\right\} .
$$

Since $A\left(\mathcal{H C}^{-}\right)=A(\mathfrak{H C})$ and $\left(G_{d}\right)^{\wedge}$ separates points, we have $\mathfrak{H}^{-}=\left(G_{d}\right)^{\wedge}$ if and only if $A(J)$ contains only the identity element of $G$, a condition equivalent to the condition that $\pi$ separates points.

1.10. Corollary. If the Abelian group $G$ admits a finite pointseparating group $\mathcal{H}$ of characters, then $G$ is finite and is isomorphic with $\mathcal{H}$.

Proof. By 1.9, we have $J_{C}=H^{-}=\left(G_{d}\right)^{\wedge}$. Since the character group of a finite group is isomorphic to the group itself, we infer that $G$ is isomorphic with $\mathrm{Je}$.

1.11. Theorem. Let $G$ be an Abelian group and let te be a pointseparating subgroup of $\left(G_{d}\right)^{\wedge}$. Then $\left(G, \mathfrak{G}_{\mathcal{J}}\right)$ is first countable if and only if He is countable.

Proof. If $\pi$ is countable, then the family of sets $U(\mathcal{F}, 1 / n)$, where $\mathscr{F}$ is a finite subset of $\mathcal{H}$ and $n>0$, is a countable base at the identity of $G$.

If there is a countable base at the identity, we may suppose that it has the form $\left\{U\left(\mathcal{F}_{n}, \varepsilon_{n}\right)\right\}_{n=1}^{\infty}$. Let $\mathcal{J}_{0}$ be the subgroup of Je gentrated by the set $\bigcup_{n=1}^{\infty} \mathcal{F}_{n}$; clearly $\mathcal{H}_{0}$ is countable. Since each $U\left(\mathcal{F}_{n}, \varepsilon_{n}\right)$ is $\mathscr{C}_{\mathcal{H}_{0}}$-open,

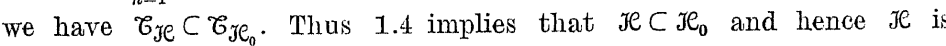
countable.

Weil's space $\bar{G}$ mentioned in 1.1 is the completion of $G$ with respect to the uniformity referred to in footnote 1 . It is obtained by the adjunction to $G$ of enough points to ensure the convergence (to a point in $\bar{G}$ ) of each Cauchy net in $G$. The fact that the group operation $(x, y) \rightarrow x y^{-1}$ from $G \times G$ into $G$ admits a continuous extension to $\bar{G} \times \bar{G}$ results from the fact that on $G \times G$ the function is uniformly continuous into the complete space $\bar{G}$.

Weil's construction of $\bar{G}$, couched entirely in the vocabulary of uniform spaces, is of necessity topological in nature; the group-theoretic properties of $G$ are, so to speak, ignored as long as possible. Restricting our attention to the case in which $G$ is totally bounded, we offer in theorem 1.12 below another characterization of $\bar{G}$.

We emphasize that our theorem does not replace or reprove Weil's result. Indeed, our proof depends directly upon the "existence" portion of Weil's theorem. 
1.12. THEOREM. Let $(G, \mathfrak{C})$ be a totally bounded Abelian group and let $\mathcal{H}=(G, \mathfrak{G})^{\wedge}$. Let $\nu$ be the mapping from $G$ into $\left(\mathcal{J}_{d}\right)^{\wedge}$ defined as follows:

$$
\nu(x)(\chi)=\chi(x) \quad \text { for } \quad \chi \text { in } \pi \text {. }
$$

Then $v$ is a topological isomorphism of $G$ onto a dense subgroup of $\left(\mathcal{H}_{d}\right)^{\wedge}$.

Proof. It is obvious that $v$ is an algebraic isomorphism. Since $v(G)$ is a point-separating group of characters on $\mathcal{H}$, it follows from 1.9 that $v(G)$ is dense in $\left(\mathscr{H}_{d}\right)^{\wedge}$. The topology $\mathcal{C}$ is by 1.2 the topology induced on $G$ by $\mathcal{H}$. Since the topology on $\left(\mathcal{H}_{d}\right)^{\wedge}$ is also induced by $\mathcal{H}, \nu$ is a topological isomorphism.

2. On a theorem of Kertész and Szele. The generalization of the Kertész-Szele theorem promised in the introduction is 2.2 below.

2.1. LEMMA. If $G$ is a topological group and $H$ is a dense subgroup of $G$ having a base at the identity of cardinality $n$, then $G$ itself has a base at the identity of cardinality $\mathrm{n}$. In particular, if $H$ is metrizable, then is metrizable.

Proof. This is a special case of the following elementary result: If $Y$ is a dense subspace of a regular topological space $X$, and if $\mathcal{B}$ is a local base in $Y$ for the point $p \in Y$, then $\left\{\operatorname{int}_{X} \mathrm{cl}_{X} U: U \in \Re\right\}$ is a local base in $X$ for $p$.

2.2. THEOREM. Any infinite Abelian group $G$ is algebraically isomorphic with a dense nondiscrete subgroup of a metrizable locally compact group $\bar{G}$.

Proof. Let $H$ be a countably infinite subgroup of $G$. Clearly there exists a countably infinite point-separating group $\mathcal{H}$ of characters on $H$. The nondiscrete totally bounded group $\left(H, \digamma_{\mathcal{H}}\right)$ is first countable by 1.11 . We now make $G$ into a topological group $(G, \mathcal{G})$ by decreeing that

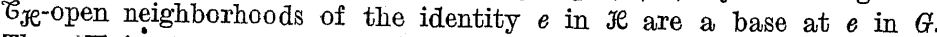
Then $H$ is $G$-open in $G$ and $(G, G)$ is a nondiscrete locally bounded metrizable group. The locally compact completion $\bar{G}$ of $(G, \mathcal{C})$ given by 1.1 is metrizable by 2.1 .

We note that if $G$ is countable, then the group $H$ of the proof above may be taken as $G$ itself, in which case $\bar{G}$ is compact.

3. Locally bounded topologies on the integers. We have already identified, at least in principle, all totally bounded group topologies on an arbitrary Abelian group $H$ : They are the topologies of the form $G_{\mathcal{H}}$, where $\mathfrak{H}$ is a dense subgroup of $\left(H_{d}\right)^{\wedge}$. For each such $\mathfrak{H}$, $\mathfrak{G}_{\mathcal{H}}$ is the topology that $H$ receives when the isomorphism $v$ given in 1.12 from $H$ into $\left(H_{d}\right)^{\wedge}$ is declared a homeomorphism. Another way to obtain all totally bounded group topologies on $H$ is to determine all compact groups containing a dense subgroup algebraically isomorphic with $H$. Sometimes such a compact group will contain many isomorphs of $H$ that are not topologically isomorphic. The following theorem, an immediate consequence of 1.1, is an aid in determining to what extent this situation may hold.

3.1. THeORem. Let $\mathfrak{G}_{1}$ and $\mathfrak{G}_{2}$ be locally compact group topologies for the Abelian group $G$. If the subgroups $H_{1}$ and $H_{2}$ of $G$ are $G_{1}$-dense and $\mathfrak{G}_{2}$-dense, respectively, and if $\tau^{\prime}$ is a topological isomorphism of $\left(H_{1}, \mathfrak{G}_{1}\right)$ onto $\left(H_{2}, \mathcal{G}_{2}\right)$, then there is a unique extension $\tau$ of $\tau^{\prime}$ that is a topotogical isomorphism of $\left(G, \mathfrak{C}_{1}\right)$ onto $\left(G, \mathfrak{G}_{2}\right)$.

We now show that the hypotheses of 3.1 may arise nontrivially. Consider the circle group $T$ with its ordinary compact topology $\zeta_{1}$. Let $\sigma$ be a discontinuous automorphism of $T$ that leaves the group $H=\left\{e^{2 \pi i r}: r\right.$ is rational\} pointwise fixed, and let $\mathfrak{C}_{2}$ be the topology on $T$ under which $\sigma$ is a homeomorphism. Then $H$ is $\mathcal{C}_{1}$-dense and $\mathfrak{C}_{2}$-dense in $G$, and the identity mapping of $\left(H, \mathfrak{C}_{1}\right)$ onto $\left(H, \mathfrak{C}_{2}\right)$ is a topological isomorphism. Note that the exteusion $\tau$ given by 3.1 is not the identity mapping on $G$; in fact, $\tau=\sigma$.

The non-algebraic analogues of theorem 3.1 and the example above for uniform spaces are given in 15.0 of [2].

3.2. Definitions. The element $y$ of the topological group $G$ will be called a topological generator of $G$ if $y$ generates a dense subgroup of $G$. A group possessing a topological generator is called a monothetic group.

We denote the group of integers by $Z$. If $y$ is a topological generator of a group $G$, we write $\mathcal{G}_{y}$ for the topology on $Z$ under which the mapping $n \rightarrow y^{n}$ is a topological isomorphism from $Z$ into $G$.

3.3. CoRoLlary (to 3.1). Let $y_{1}$ and $y_{2}$ be topological generators of the compact monothetic group $G$. Then $\mathcal{G}_{y_{1}}=\mathcal{G}_{y_{2}}$ if and only if there is a topological automorphism $\tau$ of $G$ mapping $y_{1}$ onto $y_{2}$.

Proof. For $k=1,2$, let $H_{k}=\left\{y_{k}^{n}: n \in Z\right\}$ and apply 3.1.

3.4. THEOREM. Let $y$ and $z$ be elements of $T$ having infinite order. Then $\mathfrak{G}_{z} \subset \mathfrak{G}_{y}$ if and only if $z=y^{m}$ for some integer $m$. Thus $\mathfrak{G}_{z}=\mathfrak{C}_{y}$ if and only if $y=z$ or $y=z^{-1}$.

Proof. Let $\mathcal{H}_{y}$ and $\mathcal{H}_{z}$ be the subgroups of $\left(Z_{d}\right)^{\wedge}$ generated by the characters whose values at 1 are $y$ and $z$, respectively. It is easy to see that $G_{y}=\mathscr{G}_{\mathcal{Y}}$ and $\mathfrak{G}_{z}=\mathfrak{G}_{\mathfrak{Y}}$. Since the inclusion $\mathfrak{H}_{z} \subset \mathfrak{H}_{y}$ holds if and only if $z=y^{m}$ for some integer $m$, the result follows from 1.4.

Theorem 3.4 above shows that $Z$ receives many different totally bounded group topologies from $T$. The compact group $\Delta_{p}$ of $p$-adic 
integers (defined, for example, in 10.2 of [4]) is also monothetic, but $Z$ can receive only one topology from it.

3.5. THEOREM. If $y$ and $z$ are topological generators of $\Delta_{p}$, then $\mathfrak{C}_{y}=\mathfrak{C}_{z}$.

Proof. From 10.16.a and 26.18.e of [4] one readily sees that there is a topological automorphism of $\Delta_{p}$ mapping $y$ onto $z$. Now apply 3.3 .

The topologies that $Z$ receives from $T$ and $\Delta_{p}$ are clearly 0 -dimensional. The following theorem shows that all totally bounded group topologies on $Z$ are 0 -dimensional.

3.6. THEOREM. Every totally bounded group topology on an arbitrary countable Abelian group $G$ is 0-dimensional.

Proof. The topology in question has the form $\mathfrak{C}_{\mathcal{H}}$ for some pointseparating group $\mathcal{H}$ of characters on $G$. For $\chi$ in $\mathcal{H}$, it is obvious that $\chi(G)$ is a countable subgroup of $T$. Hence there is a sequence $\left\{t_{n}(\chi)\right\}_{n=1}^{\infty}$ in $T \backslash \chi(G)$ such that $\left|1-t_{n}(\chi)\right|<1 / n$ for $n=1,2, \ldots$ It is easy to see that each set $U\left(\{\chi\},\left|1-t_{n}(\chi)\right|\right)$ is open and closed in $\mathscr{C}_{\mathfrak{Y}}$. Since

$$
\bigcap_{\chi \in \mathcal{F}} U\left(\{\chi\},\left|1-t_{n}(\chi)\right|\right) \subset U(\mathcal{F}, 1 / n)
$$

for any finite subset $\mathcal{F}$ of $\pi$ and $n>0$, the open and closed sets of the form $\bigcup_{\chi \in \mathcal{F}} U\left(\{\chi\},\left|1-t_{n}(\chi)\right|\right)$ constitute a base at the identity of $G$ for $\mho_{\mathcal{X}}$.

Added June 19, 1964. Responding to a question posed in conversation, Adam Kleppner has pointed out that the existence of non locally bounded group topologie for $Z$ has been known for many years. The following example was kindly municated to us by Shizuo Kakutani. Let $f$ be any boundet on $Z$ that is not almost periodic and yet for which the set of $\varepsilon$-translation numbers in unbounded for each $\varepsilon>0$. The expression

$$
d(m, n)=\sup _{k \in Z}|f(m+k)-f(n+k)|
$$

defines a metrizable topology of this type. That there are such functions was schown by Harald Bohr.

\section{References}

[1] M. Cotlar and R. Ricabarra, On the existence of characters in topological groups, American J. Math. 76 (1954), pp. 375-388.

[2] L. Gillman and M. Jerison, Rings of continuous functions, D. Van Nostrand Co., Princeton, 1960.

[3] J. Hejeman, Boundedness in uniform spaces and topological groups, Czechoslovak Math. J. 9 (84) (1959), pp. 544-562.

[4] E. Hewitt and K. A. Ross, Abstract harmonic analysis $I$, Springer-Verlag, Heidelberg, 1963.
[5] S. Kakutani, On cardinal numbers related with a compact abelian group, Proc. Imp. Acad. Tokyo 19 (1943), pp. 366-372.

[6] A. Kertész and T. Szele, On the existence of nondiscrete topologies in infinite Abelian groups, Publ. Math. Debr. Ecen. vol. 3 (1953), pp. 187-189.

[7] A. W eil, Sur les espaces à structure uniforme et sur la topologie générale, $\mathrm{Publ}$ Math. Univ. Strasbourg, Hermann, Paris, 1937.

THE UNIVERSITY OF ROCHESTER

Reģu par la Rédaction le 27. 5. 1963 